\title{
Population mechanisms of the green $\mathrm{Er}^{3+}: \mathrm{LiYF}_{4}$ laser
}

\author{
M. Pollnau, a) W. Lüthy, and H. P. Weber \\ Institute of Applied Physics, University of Bern, Sidlerstrasse 5, CH-3012 Bern, Switzerland
}

(Received 11 November 1994; accepted for publication 23 February 1995)

\begin{abstract}
In computer simulations the mechanisms that lead to room-temperature continuous-wave green upconversion lasing in $\mathrm{Er}^{3+}: \mathrm{LiYF}_{4}$ are investigated. The rate-equation system considers the full erbium level scheme up to ${ }^{2} H_{9 / 2}$, ground-state depletion, excited-state absorption on the pump and laser wavelengths, three interionic processes, stimulated emission, and the crystal and resonator data of the experiments. Experimental results performed at the University of Hamburg, Germany, are reproduced in the simulation. The influence of diflerent parameters as pump wavelength, absorption cross sections, interionic parameters, dopant concentration, and temperature is investigated. An avalanche effect which exploits the strong cross relaxation from the upper laser level and the upconversion from ${ }^{4} I_{13 / 2}$ leads to an efficient population of the upper laser level. At higher dopant concentrations the cross relaxation becomes detrimental to stimulated emission due to the depletion of the upper laser level. This concentration dependence can be considered as a general behavior of rare-earth-doped avalanche lasers. (1) 1995 American Institute of Physics.
\end{abstract}

\section{INTRODUCTION}

One of the interests in solid-state laser physics has focused on compact blue and green laser sources for applications in the field of data storage. A possible way toward this goal is the development of visible upconversion crystal lasers pumped by infrared laser diodes. Laser action on the green $\mathrm{Er}^{3+}$ transition ${ }^{4} S_{3 / 2} \rightarrow{ }^{4} I_{15 / 2}$ at $550 \mathrm{~nm}$ has been achieved at cryogenic temperatures in several host materials, such as $\mathrm{BaY}_{2} \mathrm{~F}_{8}, \mathrm{YAlO}_{3},{ }^{2}$ and $\mathrm{LiYF}_{4},{ }^{3,4}$ and at room temperature in fluorozirconate fibers. 5,6 Recently room-temperature lasing in $\mathrm{LiYF}_{4}$ under direct excitation ${ }^{7}$ and in an upconversion-pumped regime ${ }^{8-10}$ has been demonstrated. However, the population and deexcitation processes of the upper laser level as well as the influence of excited-state absorption (ESA) on the laser wavelength" have not yet been quantitatively understood.

In this article the results of a computer simulation of the $\mathrm{Er}^{3+}: \mathrm{LiYF}_{4}$ laser are reported. The rate-equation scheme considers all excited levels up to ${ }^{2} H_{9 / 2}$, ground-state absorption (GSA) and depletion of the ground state, ESA on the pump wavelength, three interionic processes as well as their inverse processes, stimulated emission, ESA on the laser wavelength, and the crystal and resonator data of the experiments.

From the computer simulation the population mechanisms of the laser system are quantitatively investigated. The effect of the overlap between GSA and ESA on the pump wavelength, the various impacts of the interionic processes depending on pump wavelength, the influence of ESA on the laser wavelength, as well as the concentration and temperature dependence of the output power are analyzed.

\section{SYSTEM PARAMETERS}

The labeling of the levels considers the energetic order of the $\mathrm{Er}^{3+}$ level system starting with 0 for the ground state;

${ }^{a}$ Electronic mail: pollnau iap.unibe.ch see Fig, 1. Since the ${ }^{2} H_{11 / 2}$ and ${ }^{4} S_{3 / 2}$ levels as well as the ${ }^{4} F_{5 / 2}$ and ${ }^{4} F_{3 / 2}$ levels are thermally coupled with each other they are treated as combined levels with a Bolcmannpopulation distribution. The $\mathrm{Er}^{3+}: \mathrm{LiYF}_{4}$ parameter set used in our rate-equation model is taken from Refs. 8-10 and 12-15. The intrinsic lifetimes $\tau$ of the levels are given in Fig. 1. They include radiative transitions and multiphonon relaxation. The quenching of the intrinsic lifetimes due to ion-ion interaction or ESA is considered in the rate equations. The radiative transition rates are known from a Judd-Ofelt calculation. ${ }^{12}$ The radiative transitions from ${ }^{4} S_{3 / 2}$ and ${ }^{2} H_{11 / 2}$ are weighted with their Boltzmann contribution to level 5 at $300 \mathrm{~K}$, respectively, and are summed for each transition. The same procedure was performed with the ${ }^{4} F_{5 / 2}$ and ${ }^{4} F_{3 / 2}$ levels contributing to level 7 . The nonradiative transition rates and the branching ratios are calculated in the same way as shown in Ref. 13. The important branching ratios are given in Table I(a).

Three ion-ion interactions $W_{i j}$ are included in the simulation (see Fig. 1). The parameters $W_{i j}$ of these processes for the sample doped with $1 \%$ erbium are given in Table I(b). For the parameters $W_{11}$ and $W_{22}$ a concentration dependence is experimentally observed. ${ }^{1+}$ The parameters $W_{11}$ and $W_{22}$ of the sample doped with $1 \% \mathrm{Er}^{3+}$ are assumed to have half the values measure $\mathrm{d}^{14}$ for the $10 \%$ sample. This is consistent with the results of rate-equation simulations performed in Ref. 15. The parameter $W_{50}$ is derived from our computer simulation (see Sec. IV).

The inverse processes of these ion-ion interactions are also taken into account [see Table I(b), not shown in Fig. 1]. Since for $W_{22}$ and $W_{50}$ there is a full spectral overlap between the involved manifold transitions the normal and inverse parameters are assumed to have the same values. For $W_{11}$ there is only a phonon-assisted overlap. The processes ${ }^{4} I_{13 / 2} \rightarrow{ }^{4} I_{15 / 2}$ and ${ }^{4} I_{13 / 2} \rightarrow{ }^{4} I_{9 / 2}$ have stronger transitions in the relevant area than the inverse processes due to different Boltzmann populations of the same transition in absorption 


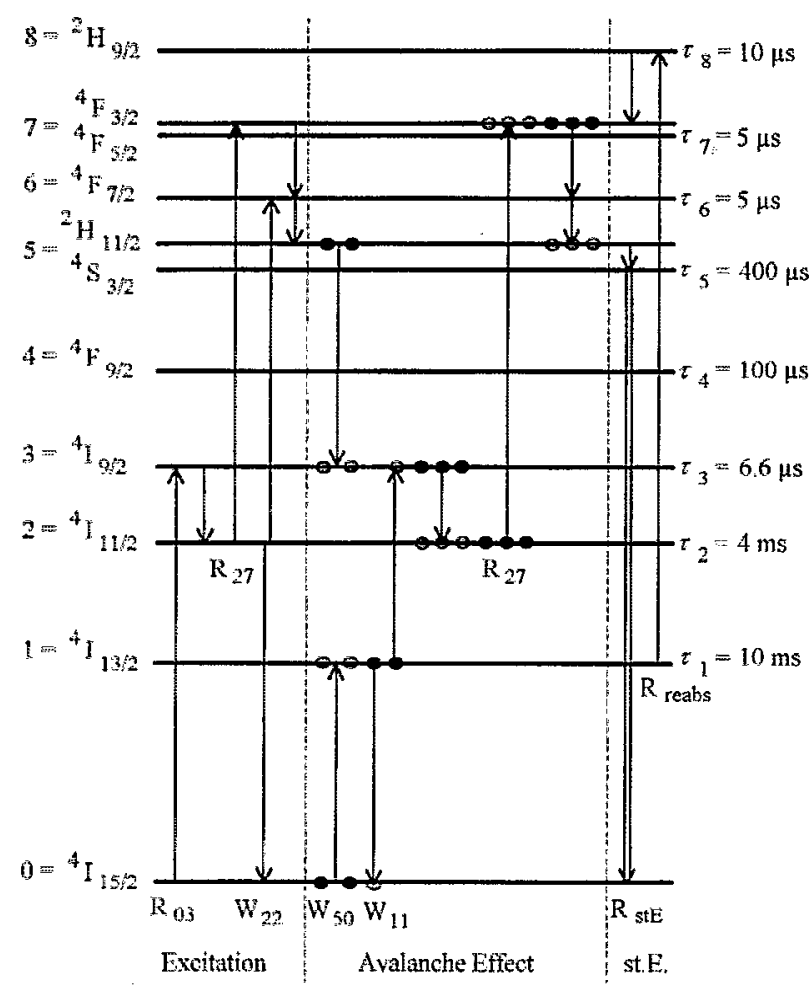

FiG. 1. Fnergy-level scheme of $\mathrm{Fr}^{3+}(1 \%): \mathrm{LiYF}$ indicating the processes that are relevant for the population and deexcitation of the ${ }^{2} S_{3 / 2}$ upper laser level at the pump wavelength $\lambda_{p}=810 \mathrm{~mm}$. (a) Excitation of the upper laser level via CSA $R_{0,3}$ and either FSA $R_{27}$ or upconversion $W_{22}$ (b) The avalanche effect including cross relaxation $W_{50}$. upconversion $W_{11}$, and ESA $R_{27}$. Solit and open dots indicate the positions of excitations before and after the tiansition, respectively, Two cross-relaxation transitions $W_{\text {so }}$, one uponversion transition $W_{11}$, and three ESA transitions $R_{2}$ are necessary to complete one avalanche cycle. The result is an enhancement of the popnlation of the upper laser level by a fator of 1.5 without pump absorption from the gromin state. Many avalanche cycles lead to the efficient population of the ${ }^{4} S_{32}$ upper laster level and to inversion on the laser transition. (c) Stimulated emission $R_{\text {s. }}$ E and reabsorption $R_{\text {reats }}$ on the laser wavelength at $551 \mathrm{~nm}$.

and emission. A brief evaluation of normal and inverse parameter considers the assistance of one phonon with energy $h \nu_{\mathrm{ph}}=400 \mathrm{~cm}^{-1}$ and includes all Stark transitions in the phonon-assisted overlap region with the same atomic cross section and the Boltomann populations of the initial levels at $300 \mathrm{~K}$. Sumuling all contributions for the normal and the inverse process results in $W_{11}=2.5 W_{30}$, which is used for the determination of $W_{30}$. With an assumption of $W_{11}=W_{30}$ the experimental results for the laser output could not be reproduced in the simulation. Experimental investigations of the parameters $W_{1 !}$ and $W_{30}$ are in progress.

The following set of parameters given by the experinnent ${ }^{6-10}$ is used in the simulation: crystal length $l=4 \mathrm{~mm}$, dopant concentration $N_{0}=1.37 \times 10^{20} \mathrm{~cm}^{-3}$ ( 1 at $\%$ on the yttrium site), a concentric resonator of optical length $l_{\text {opt }}=0.1 \mathrm{~m}$, losses due to scattering and diffraction $L_{r}=0.05$. The crystal is pumped with a Ti:sapphire laser at the pump wavelength $\lambda_{p}=810 \mathrm{~nm}{ }^{8,9}$ Experiments have also been performed at $\lambda_{p}=791 \mathrm{~nm}$ and $\lambda_{p}=974 \mathrm{~nm}$. $^{10}$ The corresponding GSA and ESA transitions and cross sections $\sigma_{i j}$ are given in Table I(c). No significant ESA from the ${ }^{4} I_{13 / 2}$ level is present at $810 \mathrm{~nm}$, whereas at $791 \mathrm{~nm}$ there is no ESA from the ${ }^{4} I_{11 / 2}$ level. ${ }^{16}$ In the experiments the following $\mathrm{cw}$ power ranges of the Ti:sapphire laser at $810 \mathrm{~nm}$ were achieved: $P_{\text {in }}<3 \mathrm{~W}$ by normal technique and $P_{\text {in }}<12 \mathrm{~W}$ with a feedback of the pump wavelength from the outcoupling mirror of the erbium cavity into the Ti:sapphire cavity." The incoupling optics transmits $\eta_{T}=0.9$ of the pump light into the erystal. The pump and the laser beam are assumed to have a radially homogeneous profile. The average radius of the laser beam within the crystal is approximately $r_{\text {mode }}=20 \mu \mathrm{m}$ and the overlap between pump and laser mode has been estimated to be $\eta_{b}=0.9$.

The laser transition starts from the second Stark level of ${ }^{4} S_{3 / 2}$ (Boltzmann population $b_{52}=0.402$ at $300 \mathrm{~K}$, degeneracy $g_{52}=2$ ) and terminates in the eighth Stark level of ${ }^{4} I_{15 / 2}\left(b_{08}=0.042, g_{08}=2\right)$ at $\lambda_{\text {laser }}=551.3 \mathrm{~nm}$. Additional reabsorption losses $R_{\text {reabs }}$ due to $\mathrm{ESA}^{11}$ from ${ }^{4} I_{13 / 2}\left(b_{11}=0.220\right.$, $\left.g_{11}=2\right)$ into ${ }^{2} H_{9 / 2}\left(b_{84}=0.142, g_{84}=2\right)$ are considered dynamically. The atomic cross sections at $551.3 \mathrm{~nm}$ and the corresponding emission and absorption cross sections are given in Table I(c). The transmission of the outcoupling mirror is $T=3 \%$.

The rate equations used in this simulation are similar to those given in Ref. 13. They additionally consider the levels $7\left({ }^{4} F_{5 / 2}+{ }^{4} F_{3 / 2}\right)$ and $8\left({ }^{2} H_{9 / 2}\right)$ as well as the ESA rate on the laser wavelength.

\section{RESULTS OF THE COMPUTER SIMULATION}

The rate equations have been calculated with the parameters given in Sec. II. The excitation mechanisms of the laser and the most important transitions for the $810 \mathrm{~nm}$ pump wavelength are indicated in Fig. 1. The upper level of the green laser emission is partly populated via two-step absorption of infrared pump photons and via upconversion from the ESA pump level (left-hand side of Fig. 1). An avalanche effect including a cross relaxation, an upconversion, and ESA leads to efficient inversion on the laser transition (central part of Fig. 1). Stimulated emission is partly reabsorbed by parasitic ESA (right-hand side of Fig. 1). These mechanisms are explained in the following section.

The dependence of the main population densities and the dominating transition rates on $810 \mathrm{~nm}$ input power can be examined in Fig. 2. The levels ${ }^{2} H_{9 / 2},{ }^{4} F_{5 / 2},{ }^{4} F_{7 / 2},{ }^{4} F_{9 / 2}$, and ${ }^{4} /{ }_{9 / 2}$ are depopulated mostly via multiphonon relaxation into the next lower-lying level; compare the branching ratios in Table I(a). Since the feeding of the ${ }^{4} F_{3 / 2}$ level via ESA from ${ }^{4} I_{1 / 2}$ is strong also the transitions ${ }^{4} F_{5 / 2}+{ }^{4} F_{3 / 2},{ }^{4} F_{7 / 2}$ and ${ }^{4} F_{7 / 2} \rightarrow{ }^{4} S_{3 / 2}+{ }^{2} H_{1 / 12}$ have significant rates which are comparable to the rate ${ }^{4} I_{11 / 2} \rightarrow{ }^{4} F_{5 / 2}+{ }^{4} F_{3 / 2}$. For simplicity these rates are not included in Fig. 2(b). All other rates have relatively small values.

The threshold and slope efficiency of the laser output significantly depend on the chosen pump wavelength. The transitions and cross sections that correspond to the experimentally and numerically investigated pump wavelengths are given in Table I(c). The input-output curves calculated in the computer simulations are shown in Fig. 3. The lowest threshold $\left(P_{\text {in }}=0.7 \mathrm{~W}\right)$ and highest slope efficiency $(1.5 \%$ versus 
TABLE I. (a) Branching ratios with a value of more than $2 \%$. (b) Interionic processes and their inverse processes, corresponding relaxation and upconversion transitions, and parameters used in the simulation. (c) Different GSA and ESA pump transitions used in the experiments (Rets. 8-10), Boltzmann-weighted punp cross sections $\sigma_{i j}$ (Ref. 15), laser and ESA on the laser wavelength, atomic cross section $\sigma_{50 j}$ at $551.3 \mathrm{~nm}$, corresponding emission and absorption cross sections $\sigma_{\mathrm{em}}=\sigma_{50} / b_{92}, \sigma_{\mathrm{abs}}=\sigma_{50} b_{98}$ (Ref. 15), and atomic cross section $\sigma_{18}$ for ESA at $551.3 \mathrm{~nm}$ calculated from Ref. 15 .

\begin{tabular}{|c|c|c|c|c|}
\hline (a) & Initial level & Branching ratio & & \\
\hline & $\begin{array}{c}{ }^{2} H_{9 / 2} \\
{ }^{4} F_{5 / 2}+{ }^{4} F_{3 / 2} \\
{ }^{4} F_{7 / 2} \\
{ }^{4} S_{3 / 2}+{ }^{2} H_{11 / 2} \\
{ }^{4} F_{y / 2} \\
{ }^{4} I_{9 / 2} \\
{ }^{4} I_{11 / 2} \\
{ }^{4} I_{13 / 2}\end{array}$ & $\begin{array}{l}\beta_{87}=0.978 \\
\beta_{76}=0.985 \\
\beta_{65}=0.941 \\
\beta_{54}=0.306 \\
\beta_{43}=0.903 \\
\beta_{32}=0.999 \\
\beta_{21}=0.387 \\
\beta_{10}=1.000\end{array}$ & $\begin{array}{l}\beta_{60}=0.051 \\
\beta_{51}=0.179 \\
\beta_{40}=0.087 \\
\beta_{20}=0.613\end{array}$ & $\beta_{50}=0.488$ \\
\hline \multirow[t]{2}{*}{ (b) } & $\begin{array}{l}\text { Interionic } \\
\text { process }\end{array}$ & $\begin{array}{l}\text { Relaxation } \\
\text { process }\end{array}$ & $\begin{array}{l}\text { Upconversion } \\
\text { process }\end{array}$ & $\begin{array}{c}\text { Parameter } \\
\left(10^{-18} \mathrm{~cm}^{3} \mathrm{~s}^{-1}\right)\end{array}$ \\
\hline & $\begin{array}{l}W_{11} \text { (normal) } \\
W_{30} \text { (inverse) } \\
W_{22} \text { (normal) } \\
W_{60} \text { (inverse) } \\
W_{50} \text { (normal) } \\
W_{13} \text { (inverse) }\end{array}$ & $\begin{array}{l}{ }^{4} I_{13 / 2} \rightarrow{ }^{4} I_{15 / 2} \\
{ }^{4} I_{9 / 2} \rightarrow{ }^{4} I_{13 / 2} \\
{ }^{4} I_{1 / 2 / 2} \rightarrow{ }^{4} I_{15 / 2} \\
{ }^{4} F_{7 / 2} \rightarrow{ }^{4} I_{1 / 2} \\
{ }^{2} H_{1 / / 2} \rightarrow{ }^{4} I_{9 / 2} \\
{ }^{4} I_{1 / 3 / 2} \rightarrow{ }^{4} I_{15 / 2}\end{array}$ & $\begin{array}{l}{ }^{4} I_{13 / 2} \rightarrow{ }^{4} I_{9 / 2} \\
{ }^{4} I_{15 / 2} \rightarrow{ }^{4} I_{13 / 2} \\
{ }^{4} I_{11 / 2} \rightarrow{ }^{4} F_{7 / 2} \\
{ }^{4} I_{15 / 2} \rightarrow{ }^{4} I_{11 / 2} \\
{ }^{4} I_{15 / 2} \rightarrow{ }^{4} I_{13 / 2} \\
{ }^{4} I_{9 / 2} \rightarrow{ }^{2} H_{11 / 2}\end{array}$ & $\begin{array}{c}W_{11}=15 \\
W_{30}=6 \\
W_{22}=9 \\
W_{60}=9 \\
W_{50}=600 \\
W_{13}=600\end{array}$ \\
\hline \multirow[t]{2}{*}{ (c) } & $\begin{array}{l}\text { Wavelength } \\
(\mathrm{nm})\end{array}$ & Process & Transition & $\begin{array}{c}\text { Cross section } \\
\left(10^{-20} \mathrm{~cm}^{2}\right)\end{array}$ \\
\hline & $\begin{array}{l}791 \\
810 \\
974\end{array}$ & $\begin{array}{l}\text { GSA } \\
\text { ESA } \\
\text { GSA } \\
\text { ESA } \\
\text { GSA } \\
\text { ESA } \\
\text { st. E }\end{array}$ & $\begin{array}{c}{ }^{4} I_{15 / 2} \rightarrow{ }^{4} I_{9 / 2} \\
{ }^{4} I_{13 / 2} \rightarrow{ }^{2} H_{11 / 2} \\
{ }^{4} I_{15 / 2} \rightarrow{ }^{4} I_{9 / 2} \\
{ }^{4} I_{11 / 2} \rightarrow{ }^{4} F_{3 / 2} \\
{ }^{4} I_{15 / 2} \rightarrow{ }^{4} I_{11 / 2} \\
{ }^{4} I_{11 / 2} \rightarrow{ }^{4} F_{7 / 2} \\
{ }^{4} S_{3 / 2} \rightarrow{ }^{4} I_{15 / 2} \\
{ }^{4} S_{3 / 2} \rightarrow{ }^{4} I_{15 / 2} \\
{ }^{4} I_{15 i 2} \rightarrow{ }^{4} S_{3 / 2} \\
{ }^{4} I_{13 / 2} \rightarrow{ }^{2} H_{5 / 2}\end{array}$ & $\begin{array}{c}\sigma_{03}=0.085 \\
\sigma_{15}=0.25 \\
\sigma_{03}=0.058 \\
\sigma_{27}=0.5 \\
\sigma_{02}=0.18 \\
\sigma_{26}=1.5 \\
\sigma_{50}=5.0 \\
\sigma_{e m}=2.0 \\
\sigma_{1 b s}=0.2 \\
\sigma_{18}=0.05\end{array}$ \\
\hline
\end{tabular}

aStimulated emission.

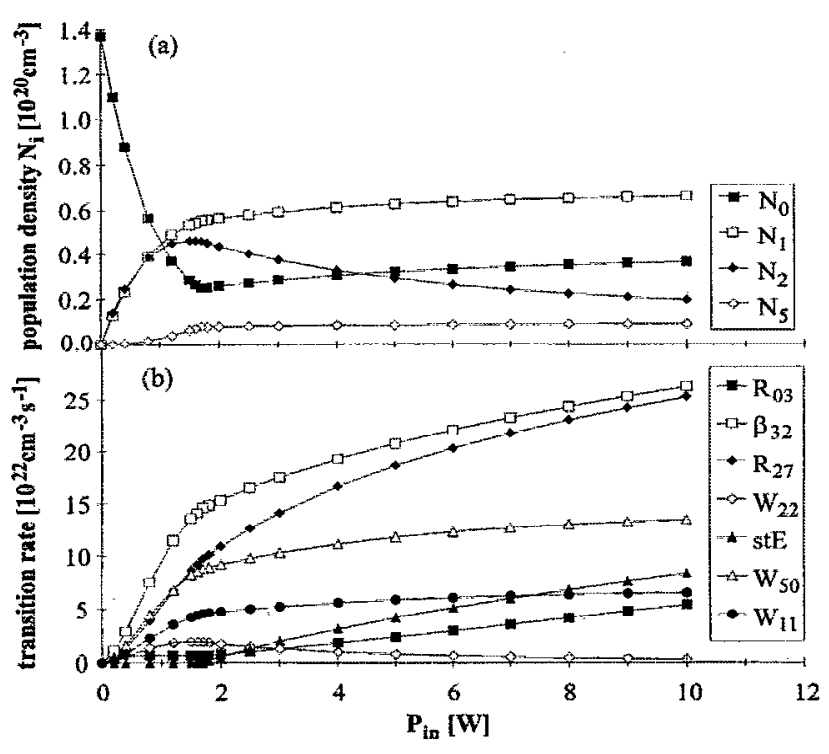

FIG. 2. Results of the computer simulations for $\mathrm{Er}^{3+}(1 \%): \mathrm{LiYF}_{4}$ with $\lambda_{p}=810 \mathrm{~nm}$ : (a) The population densities of the relevant levels; (b) the important transition rates of the system. The rate given for an interionic process is the net sum of the rates of normal and inverse process. input power at $P_{\text {in }}>4 \mathrm{~W}$ ) is achieved at $\lambda_{p}=974 \mathrm{~nm}$. The output power at $\lambda_{p}=810 \mathrm{~nm}$ is approximately three times lower. The threshold critically depends on the pump and laser beam waist. At $\lambda_{p}=810 \mathrm{~nm}$ threshold powers of $P_{\mathrm{in}}=1.8$ $\mathrm{W}$ and $1.0 \mathrm{~W}$ are calculated for $r_{\text {mode }}=20$ and $15 \mu \mathrm{m}$, respectively. This is the crucial parameter for the achievement of laser-diode-pumped $\mathrm{cw}$ lasing. The performance at $\lambda_{p}=791 \mathrm{~nm}$ exhibits a higher threshold and a smaller slope efficiency than at $\lambda_{p}=810 \mathrm{~nm}$. These calculations are in agreement with the experimental results. ${ }^{8-10}$ The effects of GSA and ESA cross sections and the influence of upconversion from the ESA pump levels are discussed in the following section.

\section{DISCUSSION OF THE POPULATION MECHANISMS}

In this section the population mechanisms of the system are analyzed. The influence of several parameters including pump wavelength, GSA and ESA cross sections, interionic upconversion and cross relaxation, dopant concentration, and temperature is investigated. 


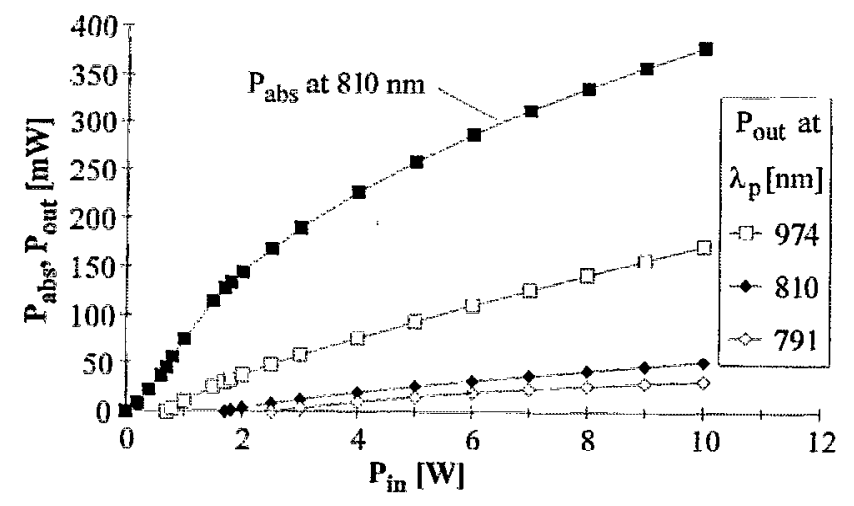

HIG. 3. Calculated input-output curves of the $551 \mathrm{~nm} \mathrm{Er}^{3+}(\mathrm{i} \%): \mathrm{LiYF}_{4}$ laser for different pump wavelengths. The beam waist is $r_{\text {nwe }}=20 \mu \mathrm{m}$ for $\lambda_{\beta}=810 \mathrm{~mm}$ and $791 \mathrm{~mm}, r_{\mathrm{mat}}=24 \mu \mathrm{m}$ for $\lambda_{p}=974 \mathrm{~mm}$. Upper curve: absorbed pump power at $\lambda_{z}=810 \mathrm{~nm}$.

\section{A. Pump excitation via GSA and ESA from the ${ }^{4} I_{11 / 2}$ level}

At the pump wavelengths 810 and $974 \mathrm{~nm}$ the ESA from the $I_{11 / 2}$ level is exploited, see Table $I(c)$ and Fig. 1. In Fig. 4 the results of the parameter variation $P_{\text {out }}\left(W_{22}, \sigma_{03}, \sigma_{27}\right)$ for $P_{\text {in }}=3 \mathrm{~W}$ at $\lambda_{p}=810 \mathrm{~nm}$ are given. The data show that the best overlap between GSA and ESA cross sections leads to the highest laser output at $551 \mathrm{~nm}$, in agreement with experiments. ${ }^{8-10}$ This explains the difference of the behavior when pumping at 810 and $974 \mathrm{~nm}$. The population mechanisms are identical except for the smaller GSA and ESA cross sections at $\lambda_{p}=810 \mathrm{~nm}$ and the additional multiphonon relaxations ${ }^{4} I_{9 / 2} \rightarrow{ }^{4} I_{11 / 2}$ and ${ }^{4} F_{5 / 2} \rightarrow{ }^{4} F_{7 / 2}$ required at this pump wavelength. These effects reduce the absorption and quantum efficiency of the green Jaser, respectively, when pumping at $810 \mathrm{~nm}$.

The pump wavelength $791 \mathrm{~nm}$ leads to different excitation mechanisms because the ESA transition starts from the ${ }^{4} I_{13 / 2}$ level. ${ }^{16}$ This situation will be explained in Sec. IV E.

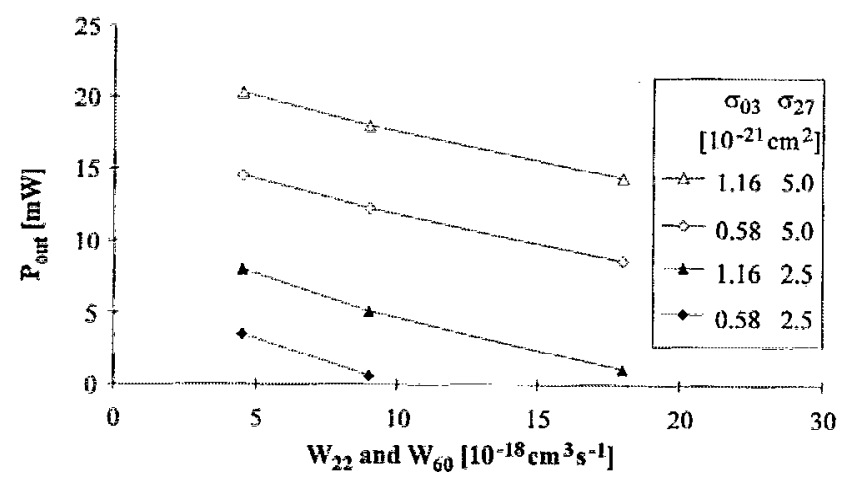

FlG. 4. Variation $P_{\text {out }}\left(W_{22}, \sigma_{13}, \sigma_{27}\right)$ for $\mathrm{Er}^{3+}(1 \%): \mathrm{LIYF}_{4}$ with $P_{\text {in }}=3 \mathrm{~W}$ at $\lambda_{\mathrm{P}}=810 \mathrm{~mm}$. The best overlap between GSA and ESA leads to the hest laser wutput. The experimental values are $\sigma_{03}=0.58 \times 10^{-21} \mathrm{~cm}^{2}, \sigma_{27}=5 \times 10^{-21}$ $\mathrm{em}^{2}$, and $W_{22}=W_{69}=9 \times 10^{-18} \mathrm{~cm}^{3} \mathrm{~s}^{-1}$. 'The upconversion $W_{22}$, although feeding the upper laser level, decreases the laser output.

\section{B. Competition of ESA and interionic upconversion}

At the pump wavelengths 810 and $974 \mathrm{~nm}$ the ESA from ${ }^{4} I_{11 / 2}$ and the upconversion $W_{22}$ populate the upper laser level ${ }^{4} S_{3 / 2}$ via multiphonon relaxation; see the left-hand side of Fig. 1 . The results of the parameter variation $P_{\text {out }}\left(W_{22}\right.$ $\left.+W_{60}, \sigma_{03}, \sigma_{27}\right)$ shown in Fig. 4 demonstrate that a stronger upconversion $W_{22}$ is decreasing the laser output, although the net sum of $W_{22}$ and $W_{60}$ populates the upper laser level. This can be explained by the following considerations. Owing to the low dopant concentration and the small GSA and ESA cross sections only a snall part of the pump power is absorbed in the crystal (see Fig. 3). An increase of the population of either the GSA or the ESA pump level would linearly increase the absorbed pump power on the corresponding transition. The upconversion $W_{22}$ removes two excitations from the ESA pump level, but transfers only one of them into the upper laser level. Without upconversion both excitations could be pumped via ESA into the upper laser level. Thus, the detrimental effect of the upconversion $W_{22}$ is the transfer of energy from the ESA pump level into the ground state which leads to the deexcitation of the system.

\section{Avalanche effect}

Since the GSA is weak the two-step excitation of the upper laser level via GSA and ESA would only lead to a small inversion on the laser transition. Therefore, other excitation mechanisms gain importance. The computer simulation gives evidence that an avalanche process strongly supports the excitation of the crystal; see the central part of Fig. 1 . The cross relaxation $W_{50}$ from the upper laser level repopulates the ESA pump level. In addition it excites the ${ }^{4} I_{13 / 2}$ level from the ground state. Half of this additional excitation is transferred into the ESA pump level by an upconversion. Although the parameter $W_{11}$ for this upconversion has a small value at $1 \% \mathrm{Er}^{3+}$ concentration, the long ${ }^{4} l_{13 / 2}$ lifetime and its high population due to the cross relaxation lead to a strong upconversion rate.

One avalanche cycle starts with two excitations in the upper laser level and finishes with three excitations in the upper laser level (sec Fig. 1), which is an enhancement factor of 1.5. At moderate pump powers this excitation channel is more efficient than the weak GSA. The laser output is nevertheless increased by a stronger GSA. This has been confirmed in the experiment ${ }^{8}$ and in the simulation (see Fig. 4).

The onset of the avalanche effect can be examined in Fig. 3. Below $1 \mathrm{~W}$ input power the absorbed pump power is rising over proportional with respect to the input power. At threshold the rate of the half part of $W_{U}$ that populates the ESA pump level is seven times higher than the rate of the GSA. The GSA rate saturates below threshold, [see Fig. 2(b)] because of the strong bleaching of the ground state; see Fig. 2(a). Above threshold the ground state is repopulated by the operating laser transition and the GSA rate is linearly increasing. The excitation via the avalanche effect, however, stagnates, because the population densities of the ${ }^{4} I_{15 / 2}$, ${ }^{4} I_{13 / 2}$, and ${ }^{4} S_{3 / 2}$ levels and, therefore, the rates of the interionic processes from these levels remain approximately con- 
stant (see Fig. 2). At $12 \mathrm{~W}$ input power the GSA rate equals the upconversion rate from ${ }^{4} l_{13 / 2}$.

\section{Decrease in slope efficiency with rising input power}

In Fig. 3 it is demonstrated that the absorbed pump power does not rise linearly with input power but shows a saturation effect. The output power is almost linearly rising with absorbed pump power. As a result the output power exhibits the same saturation effect. This decrease in the slope efficiency of absorbed pump power and laser output power is due to the depletion of the ESA pump level ${ }^{4} I_{11 / 2}$; see Fig. 2(a). Above threshold the population densities of the ${ }^{4} I_{15 / 2}$, ${ }^{4} I_{13 / 2}$, and ${ }^{4} S_{3 / 2}$ levels and, therefore, the feeding of the ESA pump level via the avalanche process saturate. The rising GSA rate cannot compensate for the depletion of the ${ }^{4} I_{11 / 2}$ level via ESA, because the GSA cross section is one order of magnitude smaller than the ESA cross section.

\section{E. Pumping at $791 \mathrm{~nm}$ by ESA from the ${ }^{4} I_{13 / 2}$ level}

At $\lambda_{n}=791 \mathrm{~nm}$ the system is pumped on the transitions ${ }^{4} I_{15 / 2} \rightarrow{ }^{4} I_{9 / 2}$ and ${ }^{4} I_{1,3 / 2} \rightarrow{ }^{2} H_{11 / 2}$. There is no ESA from ${ }^{4} I_{11 / 2}$ on this wavelength. ${ }^{16}$ With the results of the preceding subsections the small slope efficiency exhibited at $\lambda_{p}=791$ nm can be understood. Since the ${ }^{4} I_{13 / 2}$ level is highly populated under ew excitation via the cross relaxation $W_{50}$ [see Fig. 2(a)] one can expect an efficient absorption from this level. Two mechanisms are responsible for the nevertheless poor performance at this wavelength. First, the ESA cross section is considerahly lower at $791 \mathrm{~nm}$. Second, the strong upconversion $W_{11}$ from this level which completes the avalanche cycle when pumping from the ${ }^{4} I_{11 / 2}$ level becomes detrimental to stimulated emission when pumping from the ${ }^{4} I_{13 / 2}$ level. Two excitations are lost for ESA pumping. One is deexcited to the ground state and one may repopulate the ${ }^{4} I_{13 / 2}$ level after contributing to the heating of the crystal by multiphonon relaxation. Thus the positive effect of the upconversion $W_{11}$ is turned into its opposite. Its effect is worse than the detrimental effect of the upconversion $W_{22}$ when pumping from the ${ }^{4} I_{11 / 2}$ level because $W_{11}$ is stronger and it does not populate the upper laser level.

\section{F. Concentration dependence}

The cross relaxation $W_{50}$ leads to efficient avalanche lasing at low dopant concentrations. On the other hand this cross relaxation depletes the upper laser level. The effect of the cross relaxation $W_{50}$ at different dopant concentrations is shown in Fig. 5: At $1 \%$ concentration a stronger cross relaxation increases the laser output, which demonstrates the avalanche effect. At considerably higher dopant concentrations the rate of $W_{50}$ successfully competes with stimulated emission. This decreases the output power and limits the possible range of dopant concentration. The parameter that reproduces the experimental results for the laser output at $1 \%$ concentration and gives a good explanation of the experimentally observed concentration dependence is $W_{50}=W_{13}$ $=6( \pm 4) \times 10^{-22} \mathrm{~m}^{3} \mathrm{~s}^{-1}$. This is, to our knowledge, the first estimation of the cross-relaxation parameter in $\mathrm{Er}^{3+}: \mathrm{LiYF}_{4}$.

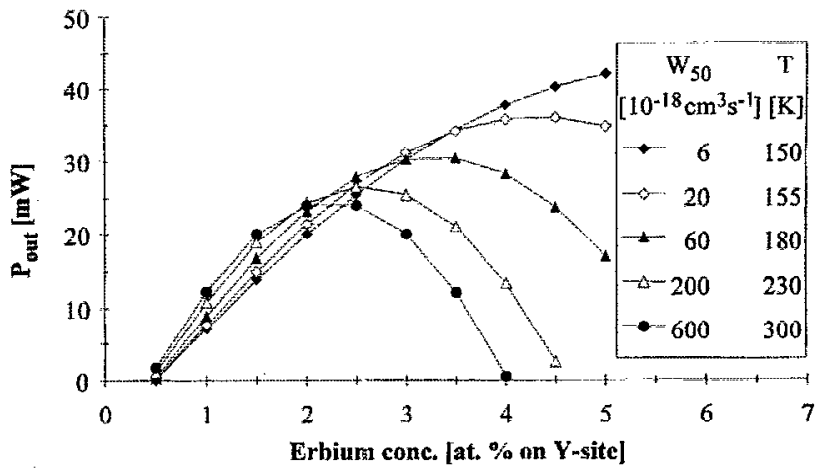

FIG. 5. The influence of dopant concentration on the laser output due to the quenching of the ${ }^{4} S_{3 / 2}$ upper laser level via cross relaxation $W_{50}$. The parameter which reproduces the experimentally observed concentration dependence is $W_{50}=600 \times 10^{-18} \mathrm{~cm}^{3} \mathrm{~s}^{-1}$. The variation of the parameter $W_{50}$ cor responds to the temperature-deperdent Boltzmann population of the ${ }^{2} H_{11 / 2}$ level.

Owing to the quenching effect of the cross relaxation a considerably higher erbium concentration cannot be used for the enhancement of the GSA and ESA. This would otherwise be useful for a better pump absorption and would increase the laser output (see Fig. 4). In rare-earth-doped crystals with a large number of energy levels in the visible range most of the lifetimes of the levels are quenched by multiphonon transitions and hardly more than one metastable level can be found in the visible energy range. The strong cross relaxation required for the avalanche effect must start from a highly populated (metastable) level. Thus, a coincidence of the initial levels of stimulated emission and cross relaxation is very likely to occur. The observed concentration dependence can, therefore, be considered as a general betavior of many avalanche lasers.

\section{G. Temperature dependence}

The results of the computer simulation are obtained with the assumption of a constant crystal temperature $T=300 \mathrm{~K}$. Thermal effects within the crystal are not included in the calculation because the crystal is not very sensitive to those effects in the experiment. It is, however, of importance to discuss the influence of temperature on the crucial parameter of the system, the cross relaxation $W_{50}$.

The data of Fig. 5 can be interpreted in two different ways. The rate corresponding to the investigated cross relaxation is given by $R_{\mathrm{CR}}=W_{50} N_{5} N_{0}$. In more detail this can be written as

$$
\dot{R}_{\mathrm{CR}}=Q_{50} b_{5 i} N_{5} b_{0 j} N_{0}
$$

Here $Q_{50}$ is the cross-relaxation parameter and $N_{i}$ are the population densities of the initial levels. $b_{5 i}$ and $b_{0 j}$ are the sum of the Boltzmann populations of all Stark levels of ${ }^{4} S_{3 / 2}+{ }^{2} H_{11 / 2}$ and ${ }^{4} I_{15 / 2}$, respectively, that contribute to the spectral overlap of emission from ${ }^{4} S_{3 / 2}+{ }^{2} H_{11 / 2}$ and absorption from ${ }^{4} I_{15 / 2}$.

In the preceding paragraph the interpretation of Fig. 5 and the given values of $W_{50}$ are hased on the assumptions $W_{50}=Q_{50}$ and $b_{5 i}=b_{0 j}=1$. The variation of $W_{50}$ can, e.g. 
correspond to a change of the host material which involves a change in the spectral overlap. The increase in concentration induces an increase of $N_{5}$ and $N_{0}$ in Eq. (1).

In this paragraph the variation of $W_{50}$ (and simultaneously $W_{13}$ ) in Fig. 5 shall reflect the temperature dependence of the cross relaxation $W_{50}$. Only the emission from ${ }^{2} H_{11 / 2}$ has a spectral overlap with the absorption from ${ }^{4} I_{15 / 2}$. The ${ }^{2} H_{11 / 2}$ level is thermally populated from ${ }^{4} S_{3 / 2}$. This involves a temperature dependence of the resonance of emission and absorption. Therefore, at room temperature the ${ }^{4} S_{3 / 2}$ lifetime is concentration quenched by the cross relaxation $W_{50}$, whereas this effect is absent at low temperatures. ${ }^{17}$ Equation (1) can be rearranged so that $W_{50}=b_{5 i} b_{0, j} Q_{50}$. The variation of $W_{50}$ in the rate equations thus corresponds to the temperature dependence of $b_{5 i}$ in Eq. (1). The value of $b_{5 i}$ decreases with decreasing temperature. The temperatures given in Fig. 5 are based on the assumption that $W_{50}$ $=W_{13}=6 \times 10^{-22} \mathrm{~m}^{3} \mathrm{~s}^{-1}$ at $300 \mathrm{~K}$ and that all Stark levels of ${ }^{2} H_{11 / 2}$ are contributing to the spectral overlap and to $b_{5 i}$ in the same way.

The temperature dependence leads to an even faster concentration quenching of the output power than calculated in the simulation. At higher dopant concentrations the higher pump absorption leads to a higher temperature within the pump channel. This increases the parameter $W_{50}$. Also the higher Boltzmann population of the terminating laser level will reduce the laser output.

\section{H. ESA on the laser wavelength}

The excited-state transition ${ }^{4} I_{13 / 2} \rightarrow{ }^{2} H_{9 / 2}$ (see Fig. 1) has a spectral overlap with the stimulated emission around $550 \mathrm{~nm}$. Since the ${ }^{4} I_{13 / 2}$ level has a long lifetime and is highly populated [see Fig. 2(a)], a significant portion of laser light may be reabsorbed on this transition. The effect depends on the spectral position of single Stark transitions and the corresponding cross sections.

It has been demonstrated that this ESA is strong enough to prevent laser action at room temperature in $\mathrm{Er}^{3+}: \mathrm{YAlO}_{3}$ because it heavily influences the long-wavelength side of the green fluorescence. ${ }^{11}$ In the host materials $\mathrm{Cs}_{3} \mathrm{Er}_{2} \mathrm{Br}_{9}$ and $\mathrm{Er}^{3+}: \mathrm{Cs}_{3} \mathrm{Lu}_{2} \mathrm{Br}_{9}$ the large size of the $\mathrm{Br}^{-}$ion leads to a smaller effect of the ligand crystal field and, therefore, a smaller Stark splitting of the spin-orbit levels. This leads to smaller wavelength ranges for all transitions. As a consequence, there is no spectral overlap for emission and ESA in these hosts. ${ }^{18}$

In $\mathrm{Er}^{3+}: \mathrm{LiYF}_{4}$ the laser line is located between two ESA peaks and the ESA cross section at $551.3 \mathrm{~nm}$ is extremely small. ${ }^{15}$ The assumption of an ESA transition with a small cross section [see Table I(c)] leads to the result that above threshold $5 \%-7 \%$ of the laser light is reabsorbed depending on pump power. This demonstrates that the small ESA on the laser wavelength affects stimulated emission in $\mathrm{Er}^{3+}: \mathrm{LiYF}_{4}$ only slightly. With $3 \mathrm{~W}$ input power at $810 \mathrm{~nm} \mathrm{cw}$ laser operation would terminate at a cross section $\sigma_{18}>1.2 \times 10^{-20}$ $\mathrm{cm}^{2}$.

\section{CONCLUSIONS}

In computer simulations the population mechanisms of the room-temperature $\mathrm{cw} \mathrm{Er}^{3+}: \mathrm{LiYF}_{4}$ laser at $551 \mathrm{~nm}$ have been analyzed. We have shown that the pump light is mainly absorbed via ESA from the ${ }^{4} I_{11 / 2}$ level into the upper laser level. The GSA transition is weak. Efficient population of the ESA pump level is achieved via an avalanche effect which exploits the strong cross relaxation from the upper laser level and the upconversion from ${ }^{4} I_{13 / 2}$. The upconversion from ${ }^{4} I_{11 / 2}$, although populating the upper laser level, is decreasing the laser output. It competes with the ESA which has twice its efficiency for the population of the upper laser level.

The experimental results for the laser output power have been reproduced in the simulation. The pump wavelength at $974 \mathrm{~nm}$ leads to the highest laser output, because GSA and ESA cross sections are favorable at this wavelength. ESA on the laser wavelength plays a minor role in $\mathrm{Er}^{3+}: \mathrm{LiYF}_{4}$ because of its small cross section at $551.3 \mathrm{~nm}$.

The cross relaxation from the ${ }^{2} H_{11 / 2}$ level is shown to be the crucial process for the green erbium laser. For the first time, the parameter for this cross relaxation and its inverse process has been determined. The value is $W_{50}$ $=W_{13}=6( \pm 4) \times 10^{-22} \mathrm{~m}^{3} \mathrm{~s}^{-1}$. A decrease of the slope efficiency with rising input power is due to the saturation of the avalanche effect above threshold and the depletion of the ESA pump level. At higher dopant concentrations the cross relaxation becomes detrimental to stimulated emission due to the depletion of the upper laser level. This concentration dependence can be considered as a general feature of rareearth-doped avalanche lasers.

\section{ACKNOWLEDGMENTS}

The authors thank Ernst Heumann of the Institute of Laser Physics, University of Hamburg, Germany, for providing the latest experimental data and for many helpful discussions. This work was supported in part by the Swiss Priority Program "Optique."

'L. F. Johnson and H. J. Guggenheim, Appl. Phys. Lett. 20, 474 (1972).

${ }^{2}$ A. J. Silversmith, W. Lenth, and R. M. Macfarlane, Appl. Phys. Lett. 51, 1977 (1987).

${ }^{3}$ P. Xie and S. C. Rand, Opt. Lett. 17, 1198 (1992).

${ }^{4}$ R. R. Stephens and R. A. McFarlane, Opt. Lett. 18, 34 (1993).

${ }^{5}$ T. J. Whitley, C. A. Millar, R. Wyatt, M. C. Brierley, and D. Szebesta, Electron. Lett. 27, 1785 (1991).

${ }^{6} \mathrm{~J}$. Y. Allen, M. Monerie, and H. Poignant, Electron. Lett. 28, 111 (1992).

${ }^{7}$ R. Brede, T. Danger, E. Heumann, G. Huber, and B. H. T. Chai, Appl. Phys. Lett. 63, 729 (1993).

${ }^{8}$ F. Heine, E. Heumann, T. Danger, T. Schweizer, J. Koetke, G. Huber, and B. Chai, OSA Proceedings on Advanced Solid-State Lasers, edited by 'T. Y. Fan and B. H. T. Chai (Optical Society of America, Washington, DC, 1994), Vol. 20, pp. 344-347.

${ }^{9}$ F. Heine, E. Heumann, T. Danger, T. Schweizer, J. Koetke, G. Huber, and B. Chai, in Conference on Lasers and Electro-Optics Europe Technical Digest, Amsterdam 1994, paper CMA6, pp. 3-4.

${ }^{10}$ F. Heine, E. Heumann, P. Möbert ${ }_{x}$ G. Huber, and B. H. T. Chai, in "Advanced Solid-State Lasers," OSA Technical. Digest (Optical Society of America, Washington, DC, 1995), pp. 267-269. 
"M. Pollnau, E. Heumann, and G. Huber, J. Lumin. 60\&61, 842 (1994).

${ }^{12}$ C. Li, Y. Guyot, C. Linarès, R. Moncorge, and M. F. Joubert, in Advanced Solid-State Lasers and Compact Blue-Green Lasers Technical Digest (Optical Society of America, Washington, DC, 1993), Vol. 2, pp. 423-425.

${ }^{13}$ M. Polnau, Th. Graf, J. E. Balmer, W. Lüthy, and H. P. Weber, Phys. Rev. A 49, 3990 (1994).

${ }^{14} \mathrm{H}$. Chou and H. P. Jenssen, in Tutable Solid State Lasers, Vol. 5 of the OSA Proceeding Series, edited by M. L. Shand and H. P. Jenssen (Optical
Society of America, Washington, DC, 1989), pp. 167-174.

${ }^{15}$ R. Brede, M.S. thesis, Institute of Laser Physics, University of Hamburg, Germany, 1993.

${ }^{16}$ M. Pollnau, E. Heumann, and G. Huber, Appl. Phys. A 54, 404 (1992).

${ }^{17}$ J. P. van der Ziel, F. W. Ostermayer, Jr., and L, G. van Uitert, Phys. Rev. B 2, $4432(1970)$.

${ }^{18}$ M. Pollnau, W. Lüthy, H. P. Weber, K. Krämer, H. U. Güdel, and R. A. McFarlane (unpublished). 